\title{
UC1 sampling plan, liquid waste storage tanks, JRC Ispra
}

\author{
Gunhild von Oertzen ${ }^{1, *}$, Olaf Nitzsche ${ }^{1}$, and Artur Hashymov ${ }^{2}$ \\ ${ }^{1}$ Brenk Systemplanung GmbH, Heider-Hof-Weg 23, 52080 Aachen, Germany \\ 2 Energorisk Ltd., 141, No7, Simyi Steshenkiv str., Kiev, Ukraine
}

Received: 21 June 2019 / Received in final form: 23 September 2019 / Accepted: 9 October 2019

\begin{abstract}
The objective of INSIDER work package 3 (WP 3) is to draft a sampling guide for initial nuclear site characterization in constrained environments, based on a statistical approach. In this paper, deliverable 3.4 (D 3.4) is presented for WP 3, where the strategy developed in deliverables 3.1 (D 3.1) to 3.3 (D 3.3) is applied to the first of three reference use cases representative of existing decommissioning scenarios. The present discussion focuses on use case 1 (UC1): the liquid waste storage facility at the JRC site of Ispra (Italy). The proposed characterization strategy developed in D 3.2 is applied in a step by step approach to analyse the pre-existing information (obtained through the use of a pre-sampling questionnaire), and to utilise the available inputs towards the development of a sampling plan sufficient for allowing radiological characterization. The proposed sampling plan follows a three-step approach, i.e. determination of possible elevation in activity concentration by non-destructive testing, biased sampling of layers identified, and finally unbiased sampling after mixing of tank contents.
\end{abstract}

\section{Introduction}

\subsection{Background}

The facility selected for the case study $\mathrm{UC} 1$ is the liquid waste storage facility at the JRC site of Ispra (Italy), referred to as "tank farm". This is a building commissioned in 2010, designed to collect all remaining liquid waste present on site, mostly stored in tanks in the old liquid effluent treatment station (STRRL), to be routed for cementation or other solidification and conditioning treatment. Most of the liquid waste or sludge is contained in two double walled tanks of $12 \mathrm{~mm}$ total wall thickness, called VA001 and VA002. A small leadshielded tank for ILW was added to the storage facility a couple of years later. The latter is explicitly excluded from the sampling plan to be established for this exercise, but may contribute to the overall dose rate in the building.

The exercise is designed to build upon the sampling strategy developed for the project Improved Nuclear SIte characterization for waste minimization in DD operations under constrained EnviRonment (INSIDER), see [1]. Information for the benchmarking of the use case concept was provided by [2].

\footnotetext{
* e-mail: g.vonoertzen@brenk.com
}

\subsection{Pre-characterisation questionnaire}

A pre-characterisation questionnaire was used to determine the historical background, scope, purpose and end points of the characterisation. This was sent to the Ispra team for completion and information gathering.

From the completed questionnaire and preliminary data provided, some information is available to support in the preparation of the sampling plan:

- The historical origin of the waste is the operation of a nuclear research facility including a nuclear research reactor. No end date and further specifics of the research facility are provided.

- The stated objective of the sampling plan is to classify and characterise the waste in view of conditioning and management of the waste for storage and/or disposal, and to obtain a better understanding of the radiological safety implications of storing and processing the waste. - Apart from one sampling campaign during which the chemical and radiological properties of the tank contents were measured in 2013, no additional data from environmental or radiological surveillance relating to the waste is available.

- A stated uncertainty relates to the relative inhomogeneity of distribution of radionuclides in the waste.

- Material data safety sheets about the waste do not exist, in particular no indication of the chemical toxicity is present. 
- According to the pre-sampling questionnaire, two sets of scaling factors are available, but these have not been provided together with the sampling reports: it is a stated intent of this exercise to come up with scaling factors (if any) which are not prejudiced by way of information supplied in advance.

- The maximum dose rate on contact of the tanks is recorded to be $30 \mu \mathrm{Sv} / \mathrm{h}$. It is not clear which of the two tanks is associated with the maximum dose rate.

- Accessibility of the waste in the tanks for laboratory sampling is limited to in-stream sampling while pumping contents from the tanks or through a sampling loop.

- External access to the tanks for dose rate measurements is possible in general but is restricted because of the location of the tanks against the building walls, and by a shielding wall covering part of one tank.

- Surface contamination is not expected to be an issue here as the waste is contained within the tanks. The absence of surface contamination is stated as part of the information provided.

\section{Objectives}

\subsection{Main objective}

The main objective here is to fulfil the necessary requirements for conditioning and removing the waste according to the relevant waste acceptance and possibly disposal criteria.

It is noted that relevant waste acceptance criteria for this waste have not yet been determined by the relevant authority, and applicable clearance or acceptance limits are therefore not known. The intended treatment or conditioning strategy is still in the process of being defined; while cementation has been investigated as a possibility, this has yet to be confirmed.

Given this background, the primary objective for this campaign is to characterize the waste as exactly as possible, both in relation to its physico-chemical properties as well as to its radiological content.

\subsection{Highest priority objective}

Given the pre-existing knowledge about the waste, including the pre-existing laboratory data with comprehensive analysis of physico-chemical parameters, the radiological characterisation of the waste here is the highest priority objective, i.e. determination of type, isotopic composition and volumetric distribution of radioactive waste in waste containers (tanks) and difficult-to-measure (DTM) nuclides and their correlations or scaling factors to easy-to-measure nuclides.

\subsection{Statistical indicators}

If a uniform distribution of data is expected, an unbiased survey is the preferred sampling method, and vice versa, if the distribution is expected to be non-uniform, a biased survey is the better option. The validity and usefulness of scaling factors must be determined, using as a starting point the pre-existing data as an input. While preliminary scaling factors for specific process streams at Ispra have been determined in the past, these have not been provided so as not to prejudice the analysis of the preexisting data.

\section{Constraints}

\subsection{Access for sampling}

According to the information provided on the tanks, access for non-destructive in situ dose rate measurements and in situ gamma spectrometry is limited due to the presence of a shielding wall and due to the location of the tanks against the building walls and on the floor of the building.

In addition, destructive sampling of the sludge in the tanks is restricted to transfers of contents from the tanks to a temporary storage container, or to sampling in-stream while pumping the tank contents through a loop, via a bagin bag-out glove box arrangement.

It is noted that the historical origin of the two tanks' contents is not the same, and that mixing between the tanks does not take place. The two tanks are therefore to be characterized as separate entities.

\subsection{Homogenisation of tank contents}

Both tanks are equipped with stirrers to ensure homogeneity of the contents. It can therefore be expected that measurements before and after stirring events may yield different outcomes. Notwithstanding the option of stirring tank contents, deposition of solids at the tank bottom may have occurred that is not possible to mobilise through stirring, leading to a layered structure of the radioactive waste in the tanks.

\subsection{Reference samples}

As stated in the benchmarking design by [2], one of the major problems in characterising tanks containing sludge (mixed liquid/solid) is the unavailability of suitable reference samples with adequate solid fraction.

\section{Pre-existing data}

\subsection{Historical information}

According to the Ispra brochure [3] and additional information provided, the liquid waste to be characterized at the tank farm derives from cleaning processes during the decommissioning of research \& development projects at the research reactor. The waste consists of two tanks, each about $50 \mathrm{~m}^{3}$ in volume, of LLW sludge with activities up to a little over $100 \mathrm{~Bq} / \mathrm{g}$, and stemming from the liquid effluent treatment facility.

According to the information supplied, there is no contamination in the building; hence surface contamination measurements are not required. The maximum dose 
rate on contact on the tanks is reported to be $30 \mu \mathrm{Sv} / \mathrm{h}$. It is supposed that radiation from the ILW liquid tank located in the same building is not contributing significantly to the dose rate at the surface of tanks VA001 and VA002.

It is noted that the pre-existing samples were drawn immediately following the filling of the two tanks, and after stirring the contents. Consequently, water content of the samples is high as there has been no separation of solids within the tank contents. It is noted further that in the meantime, deposition from the sludge may have occurred on the tank bottoms, as evidenced by taking gamma dose rate readings from the tank exteriors. Further sampling at the present stage is therefore likely to result in deviations from the data found during the first sampling campaign.

\subsection{Data collected}

A set of samples was collected in the years 2012/2013, a first set consisting of 12 sludge samples from tank VA002 (referred to as WP 03, see [4]), and a second set of 12 sludge samples from tank VA001 (labelled WP 04, see [5]).

\section{Preliminary data analysis}

\subsection{Pre-processing}

An investigation of the data at hand suggests a priori no obvious errors or need for removal of individual data points. Outliers are not apparent, and the relative homogeneity of the (physico-chemical) data suggests that the samples are representative, at least for tank VA002. For tank VA001, distributions of nuclide activities displayed higher variability, likely necessitating additional sampling.

Based on the pre-existing data, it can be expected that there is some spatial non-uniformity, which is principally related to the separation into liquid and sludge/solid portions of the waste. If possible, a gamma imaging camera or alternatively gamma scanning can be utilised to identify hot spots or layering inside the tanks.

Given the small number of samples and relative inhomogeneity within the activity concentrations, the use of scaling factors in the samples for the determination of DTM nuclides can be expected to be of limited use only, if at all. Hence, a combination of biased (to confirm distribution of inhomogeneity) and unbiased (to confirm averages for overall characterisation) survey methods based on a small number of samples could be sufficient.

\subsection{Exploratory data analysis}

In this exploratory analysis, we take stock of the information available, and whether additional information is needed to reach the stated objectives.

As the time elapsed since waste generation is more than 10 years, probably significantly so, very short living nuclides are no longer expected to be present.

Apart from the radiological characteristics, the preexisting data seem to give a representative indication of a fairly homogenous physico-chemical content of the tanks, where this information is available. The parameters included in the preliminary analysis include particle size distribution, elemental composition and thermogravimetric analysis (tank VA001 only), and chemical analysis including $\mathrm{pH}$, water content, bulk density, conductivity, total solids, total suspended solids, total dissolved solids, total content of carbon and phosphorous, $\mathrm{NH}_{4}{ }^{+}$, total tensioactives, fluoride, chloride, bromide, phosphate, sulphate, nitrate, cyanide, formate, oxalate, acetate, citrate, and anionic, non-ionic and cationic tensioactives.

The relative homogeneity relating to physico-chemical content confirms the initial assessment of the highest priority objective, i.e. the determination of the spatial distribution (if any) and nuclide vector of the radionuclide content of the tanks.

\subsection{Data analysis}

In order to better understand the information available from the pre-existing data and the gaps remaining, trends relating to the radionuclide content are here further analysed.

\subsection{Post-processing}

Inspection of the pre-existing data allows the following observations to be made:

- The activity concentration of alpha-emitting nuclides (Am-241, Pu isotopes, Cm-244) is on the order of $10 \mathrm{~Bq} / \mathrm{g}$ in both tanks. Hence the initial assessment of the waste as class LLW is confirmed and further analysis of this classification is not necessary.

- The activity concentrations in the two tanks are not significantly different with ratios for individual nuclides ranging between some tenths to some tens.

- The nuclide activity concentration in tank VA001 is not representative of a homogeneous distribution, with standard deviations of the activities of up to $200 \%$. In tank VA002, the homogeneity is significantly higher, with standard deviations not exceeding some $20 \%$ for the activities.

- No information is available about the elevation level within the tank from which sampling occurred.

- The activity concentration in the samples is dominated by fission products. The ratio of Sr-90 to Cs-137 activities is very high (around 0.5 to 0.6 in both tanks, while usually a ratio between 0.1 and 0.01 is more typical). In reference [6], typical scaling factors for Italian nuclear power plants are listed, however they refer only to the four commercial nuclear reactors, two of the BWR type and one of the PWR type and gas graphite type each. The example mentioned here, the ratio of Sr-90/Cs-137 activities, is less than 0.06 in resins from $\mathrm{BWR}$ and $\mathrm{PWR}$ reactors listed in that IAEA reference.

- In both tanks there is very little solid material present, but nevertheless the $\alpha \beta / \gamma$ ratio is high compared to standard reactors (PWR, BWR). Tank VA002 probably contains more solids (95\% water) compared to VA001 (99\%). Nevertheless the $\alpha \beta / \gamma$ ratio in VA001 seems to be 
larger than in VA002. Alpha emitting nuclides are expected to be more present in the solid due to their low solubility. In summary, both tanks have a relatively high $\alpha \beta / \gamma$ ratio when compared with standard reactors. The higher $\alpha \beta / \gamma$ ratio combined with higher Sr-90/Cs-137 can also be found in spent fuel residues.

- For a statistically valid application of scaling factors, more data of DTM nuclide activities will be needed for both tanks. For tank VA001, the applicability of scaling factors is severely limited by the variability in activity concentrations, while for tank VA002, scaling factors based on the Co-60 and Cs-137 activities were of limited applicability.

- In the existing data, many of the activities of DTM nuclides were below the detection limit. However, valid characterisation of the nuclide vectors will require more information about the homogeneity, and of the usefulness of applicable scaling factors. More data will therefore be needed, including of DTM nuclides.

\subsection{Achievement of the objectives}

The pre-existing data provide comprehensive information about physico-chemical properties of the waste. Only a small number of additional samples (e.g. 6 per tank to allow for a minimum of statistical analysis) should suffice to confirm the characteristics determined via the pre-existing data, including the chemical characteristics.

In the context of historical information, it is known that the sampling was conducted immediately following tank filling and mixing; hence information about an elevation profile in activity concentrations or sludge/liquid separation is not available from that data set, and additional sampling will need to verify if such a profile exists in the tanks.

Based on the pre-existing data, the radionuclide inventory of the tanks is less homogeneous than the chemical content $(\mathrm{pH}$, water content, bulk density, conductivity, total solids, total suspended solids, total dissolved solids, total content of carbon and phosphorous, $\mathrm{NH}_{4}{ }^{+}$, total tensioactives, fluoride, chloride, bromide, phosphate, sulphate, nitrate, cyanide, formate, oxalate, acetate, citrate, and anionic, non-ionic and cationic tensioactives), which also suggests additional data will be needed to obtain a statistically valid radionuclide inventory and, if possible, relevant scaling factors.

The objectives relating to variability and nuclide content of the radioactivity in the two tanks are therefore not adequately addressed by the pre-existing data, and more sampling (both non-destructive and destructive) will have to be performed.

\section{Sampling plan - design}

\subsection{Non-destructive testing to determine possible elevation profile in activity}

The first step in the sampling campaign should be the establishment of the approximate distribution of the activity in the tanks by external gamma spectrometry or by collimated dose rate measurements. The prerogative here would be to determine if there is an elevation profile in activity concentration within the tanks, for example as a result of solids with more significant radionuclide content settling to the bottom of the tanks, hence this campaign should be performed prior to mixing, and after allowing as long a settling time as possible. This is likely to give an indication of the separation within the tanks between liquid and sludge portions of the waste, and therefore also allow an estimate of the respective quantities of sludge and liquid present.

The pre-existing data of tank VA001 displayed relative radiological inhomogeneity - this inhomogeneity may be related to sampling different portions of the sludge without adequate mixing. Non-destructive testing from the tank exterior may be useful to determine if this is the case in the present situation, i.e. if there is an elevation profile with differing activity concentrations. This is particularly relevant as at present, several years after the first samples were collected, stratification or deposition may have occurred.

For tank VA002, the sampling data suggested better homogeneity between the samples. Nevertheless, the same technique should be used to determine whether an elevation profile can be determined exterior to the tank prior to mixing.

\subsection{Biased sampling, prior to mixing}

Following non-destructive gamma dose rate measurements, there will be an indication of whether the contents are fairly homogeneous with respect to specific activity, or whether there is a significant elevation profile.

In case of an elevation profile, biased sampling should be performed on that portion of the waste with the highest activity contained, prior to performing any mixing. The number of samples to take may be limited by access of the different levels within the tank, but a minimum number of samples of about 6 may be sufficient for confirming the usefulness and applicability of previously identified scaling factors. At the same time, biased sampling along the entire height of the waste volume should provide the samples required to determine the chemical characteristics of the liquid and sludge portions of the waste, including their variability within the tanks.

A sample number of 6 provides the best compromise as an increase in samples only marginally increases the confidence interval of the standard deviation, while a smaller sample size provides insufficient statistical power.

\subsection{Unbiased sampling, following mixing}

In case of no elevation profile, sampling can skip the previous step (biased sampling) and can proceed to unbiased sampling, which should be performed after mixing tank contents. If possible, unbiased sampling should be performed in a way as to ensure that any part of the tank contents is equally likely to be sampled. 
Therefore, only the probabilistic sampling method can now be used for sampling the sludge. To ensure a valid random sampling campaign for the entire volume, it has to be ensured that the entire mobilisable volume of the tank is circulated during the sampling campaign and that samples are taken from (nearly) equal volumes from the whole stream.

The minimum number of samples is determined by the requirements of an approach for univariate statistics on non-spatially distributed data. As preparatory homogenisation measures will be used for homogenisation, the expected variance of activity concentration should be low. Therefore, the number of samples can be low (10-20).

If step 6.2 was skipped, the number of samples of this step will need to be sufficient for determination of scaling factors and range of nuclide factors. If data were collected for step 6.2 (biased sampling), the number of samples still required for the unbiased sampling step can be correspondingly reduced, as the biased sampling data can provide some information about the results to be expected after mixing.

Statistic evaluation of results will be done concerning univariate analysis only with respect to the nuclide specific activity concentration. In addition, the scaling factors of DTM to Cs-137 will be evaluated or confirmed.

Based on the pre-existing data, it can be expected (but needs confirmation) that no activity elevation profile can be found for tank VA002, while for tank VA001, an elevation profile is likely but also needs confirmation. If an elevation profile exists, biased sampling will confirm this, and the sampling data can be contributed to the data set used for characterization. If no elevation profile is identified, non-biased sampling only will need to provide sufficient data for characterization.

\subsection{Number of samples}

How many samples are required?

According to [7], the simplest approach for a univariate problem with no spatial structure is to use the standard formula

$$
n \geq\left(\frac{z \sigma}{e}\right)^{2}
$$

where $n$ is the number of samples, $z$ is the confidence level, $\sigma$ is the sample standard deviation and $e$ the error level. It is clear that the number of samples increases rapidly with the standard deviation, i.e. with the variability of the sample set. For the data set for tank VA001, values for the standard deviation in activity concentration ranged between $16 \%$ and almost $200 \%$ of the mean. Assuming a confidence level of $90 \%$ (i.e. $z=1.6$ ) and an error of $5 \%$ of the mean, the number of samples required from this set is still at least 20, when assuming the lowest value for the standard deviation found (16\% for Co-60). For the sample set from tank VA002, the situation is slightly more advantageous, as the variability in the data was smaller.
For this set, the number of samples required is at least 5 , and up to about 60 for the nuclides for which the activity concentration displayed larger variability.

In a first step therefore, it is advisable to confirm the degree of variability in the data before deciding on the number of samples required. In the case of a spatial structure being present (as determined in step 6.1), biased sampling of individual layers will lead to more homogeneous subsets for sampling, which require a smaller number of samples. As a starting point, 6 samples should be sufficient to confirm variability, allow for a minimum of statistical analysis and inform the way forward.

\section{Conclusion}

Following the guideline set out in D3.2 Report on statistical approach, see [1], we attempt here to follow the proposed strategy by applying it to the characterisation of the Ispra storage tanks.

The amount of effort needed for the sampling and characterization campaign hinges on the availability of information prior to the campaign. Information about the historical origin of the waste and the analysis of preexisting data can significantly reduce the subsequent sampling required.

The two ILW tanks at Ispra are characterized by activity concentrations of gamma emitting nuclides of a few $\mathrm{Bq} / \mathrm{g}$ (tank VA001) up to about $135 \mathrm{~Bq} / \mathrm{g}$ (tank VA002). Non-destructive gamma spectrometry from the tank surface may be used to determine if elevation profiles in the tank can be identified prior to mixing.

Biased sampling may be used to confirm inhomogeneity within the tanks, if suggested by non-destructive testing. If not, non-biased sampling only can be used to confirm the trends observed in the pre-existing data, and to supplement it where additional information is needed.

\section{Author contribution statement}

All authors contributed to the development of the sampling plan. The writing of the article was coordinated by Gunhild von Oertzen.

\section{References}

1. B. Rogiers, S. Boden, N. Perot, Y. Desnoyers, O. Sevbo, O. Nitzsche, Report on statistical approach, INSIDER WP3Sampling strategy, Deliverable D3.2, 2018

2. P. Peerani, S. Boden, M. Crozet, F. Zanovello, M. Herranz, Design of the benchmarking exercise, INSIDER WP2Requirement and validation, Deliverable D2.5, 2017

3. European Commission, Nuclear Decommissioning and Waste Management Programme at the Joint Research Centre, Ispra Site, Joint Research Centre, 2016

4. P. Londyn (ENVINET), Final Chemical and Radiological Analysis Report, Work Package 03-2011, 2013

5. P. Londyn (ENVINET), Final Chemical and Radiological Analysis Report, Work Package 04-2012, 2013 
6. International Atomic Energy Agency, Determination and use of scaling factors for waste characterization in nuclear power plants, IAEA Nuclear Energy Series No. NW-T-1.18, IAEA, Vienna, 2009
7. N. Pérot, Y. Desnoyers, G. Augé, F. Aspe, S. Boden, B. Rogiers, O. Sevbo, O. Nitsche, INSIDER WP3-Sampling strategy report on the state of the art, Deliverable 3.1, 2017

Cite this article as: Gunhild von Oertzen, Olaf Nitzsche, Artur Hashymov, UC1 sampling plan, liquid waste storage tanks, JRC Ispra, EPJ Nuclear Sci. Technol. 6, 15 (2020) 